\title{
$P$-RECURRENCE IN TOPOLOGICAL DYNAMICS ${ }^{1}$
}

\author{
JOHN D. BAUM
}

It is the purpose of this paper to extend the notions of recurrence, both positive and negative [3, Chap. 7], ${ }^{2}$ to transformation groups whose phase groups are more general than the integers or the reals. The notion of regional recurrence is similarly treated and various applications to other topics of topological dynamics are given.

1. Definitions. The general reference for definitions is [3]. Throughout the paper $(X, T, \pi)$ will denote a transformation group for which $X$ is a compact Hausdorff space. Whenever it is stated that $X$ is a uniform space, it will be implicitly assumed that the (Hausdorff) topology of $X$ is the one induced by the uniformity. It is further assumed that $T$ is abelian. $P$ and $Q$ will be used to denote replete semigroups in $T$, which are distinct from $T$. Full use of all these hypotheses is not required in every theorem. For $x \in X$ the $P$-limit set of $x$, $P_{x}$, is defined as in $[1,2.06]$. Let $E \subset T$, then $E$ is $P$-extensive provided $E \cap p P \neq \varnothing$ for each $p \in P$. Let $x \in X$, then $x$ is $\{P$-recurrent $\}$ $\{P \text {-regionally recurrent }\}^{3}$ provided that for each neighborhood, $U$, of $x$ there exists a $P$-extensive set $E$ in $T$ such that $r \in E$ implies $\{x r \in U\}\{U r \cap U \neq \varnothing\}$. It is now clear that the restriction $P \neq T$ is justified, for if we were to allow equality it would follow that each point $x \in X$ is $T$-recurrent. This would make $P$-recurrence too weak a property to be of much interest. It is also clear that a point $x \in X$ is recurrent if and only if $x$ is $P$-recurrent for each replete semigroup $P$ in $T$. A similar statement holds for regional recurrence. $T$ is said to be $\{P$-regionally recurrent $\}$ \{regionally recurrent $\}$ provided that for each $x \in X, x$ is $\{P$-regionally recurrent $\}$ \{regionally recurrent $\}$.

\section{2. $P$-recurrence.}

Lemma 1. Let $x \in X$, let $P$ be a replete semigroup in $T$, then the following statements are pairwise equivalent:

Presented to the Society, April 21, 1956; received by the editors January 17, 1955 and, in revised form, December 27, 1955.

1 This work was supported by the National Science Foundation research grant NSF-G1225.

2 Numbers in brackets refer to the bibliography.

3 Throughout this paper braces are used to indicate alternative readings of the same definition or theorem. All words in the first sets of braces are to be read together, all in the second sets together, etc. 
(1) $x$ is $P$-recurrent, i.e. if $U$ is a neighborhood of $x$, then there exists a $P$-extensive set $E \subset T$ such that $x E \subset U$.

(2) $x \in P_{x}$.

(3) $\mathrm{Cl}(x P)=P_{x} .[N . B . \mathrm{Cl}(A)$ denotes the closure of the set $A$.

(4) $\mathrm{Cl}(x T)=\mathrm{Cl}(x P)$.

(5) If $U$ is a neighborhood of $x$ and if $p \in P$, then $x p P \cap U \neq \varnothing$.

(6) If $U$ is a neighborhood of $x$ and if $t \in T$, then $x t P \cap U \neq \varnothing$.

(7) If $U$ is a neighborhood of $x$ then $x T \subset U P^{-1}$.

(8) $x \in \mathrm{Cl}(x p P)$ for each $p \in P$.

(9) $x \in \mathrm{Cl}(x t P)$ for each $t \in T$.

(10) $x$ is $p P$-recurrent for each $p \in P$.

The proof is straightforward and is omitted. It is clear that if $x \in X$ and if $P$ and $Q$ are replete semigroups in $T$ such that $P \subset Q$, then $x$ is $\{P$-recurrent $\} \quad\{P$-regionally recurrent $\}$ implies $x$ is $\{Q$ recurrent $\}$ \{ $Q$-regionally recurrent $\}$.

TheOREM 1. Let $x \in X$ and let $x$ be P-recurrent, then $x t$ is $P$-recurrent for each $t \in T$.

Proof. $x \in P_{x}$ by Lemma 1. Thus $x t \in P_{x} t=P_{x t}$ by $[3,6.34]$, and again by Lemma $1, x t$ is $P$-recurrent. This completes the proof.

We remark that if $x$ is recurrent, then for any two replete semigroups $P$ and $Q$ in $T, P_{x}=Q_{x}$; however, the converse proposition is false, for consider the point $y=\cdots 00100 \cdots$ in the bisequence space [3, Chap. 12]. Let $X=\mathrm{Cl}(y I), I$ the integers, then clearly $X=y I \cup z$, where $z=\cdots 00000 \cdots$, and $P_{x}=z$ for each $x \in X$ and each replete semigroup $P$ in $I$. We observe, however, that $y$ is not recurrent.

In case $T$ is generative $[1,1.01]$ and separable there is an intimate connection between $P$-recurrence and $P$-asymptoticity $[1,2.03]$, namely, the following statements are pairwise equivalent: (1) $x$ is not $P$-recurrent, (2) $x \notin P_{x}$, (3) $x \uparrow_{P} P_{x}$, i.e. $x$ is $P$-asymptotic to $P_{x}$.

It is known $[3,6.14]$ that if $S$ is a closed syndetic subgroup of $T$ and $P$ a replete semigroup in $T$, then $P \cap S$ is a replete semigroup in $S$. The following lemma asserts something more.

Lemma 2. Let $S$ be a closed syndetic subgroup of $T$, let $P$ be a replete semigroup in $T$, then $P \neq T$ if and only if $P \cap S \neq S$.

Proof. The sufficiency is obvious. We show the necessity. Assume the contrary, i.e. $P \cap S=S$ and $P \neq T$. Let $K \subset T$ be compact such that $T=S K$. Since $K$ is compact, there exists $t \in T$ such that $t K \subset P$. Thus $P \supset P P \supset t K P \supset t K S \supset t T \supset T$, whence $P=T$, contrary to hypothesis. This completes the proof. 
TheOREm 2. Let $S$ be a closed syndetic subgroup in $T$, let $P$ be a replete semigroup in $T$, then $E \subset S$ is $P \cap S$-extensive if and only if $E$ is $P$-extensive.

Proof. Let $E \subset S$ be $P$-extensive in $T$; then $E \cap p P \neq \varnothing$ for each $p \in P$. Then certainly $E \cap p(P \cap S) \neq \varnothing$ for each $p \in P \cap S$, whence $E$ is $P \cap S$-extensive.

Conversely, let $E \subset S$ be $P \cap S$-extensive in $S$, i.e. $E \cap p(P \cap S) \neq \varnothing$ for each $p \in P \cap S$. Let $q \in P$ and let $K \subset T$ be compact such that $T=S K$; then there exists $t \in T$ such that $t K \subset P^{-1}$. Thus StK=SKt $=T t=T$, and we may assume that $K \subset P^{-1}$. Since $q \in T, q=s p^{-1}$, where $s \in S$ and $p^{-1} \in K \subset P^{-1}$, whence $s=q p$. Now $\{s, e\}$ is a compact set in $S$, therefore there exists $r \in S$ such that $\{r s, r\} \subset P \cap S$. Then $E \cap r s(P \cap S) \neq \varnothing$ and a fortiori $E \cap r s P=E \cap r q p P \neq \varnothing$, whence $E \cap q P \supset E \cap q(r p P) \neq \varnothing$. Thus $E$ is $P$-extensive in $T$. This completes the proof.

TheOREM 3 (InHeritance theorem). Let $S$ be $a$ closed syndetic subgroup in $T$, let $T$ be locally compact, and let $P$ be a replete semigroup in $T$ then:

(1) if $x \in X, x$ is $P \cap S$-recurrent if and only if $x$ is $P$-recurrent.

(2) $S$ is pointwise $P \cap S$-recurrent if and only if $T$ is pointwise $P$ recurrent, i.e. each $x \in X$ is $P \cap S$-recurrent if and only if each $x$ is $P$ recurrent.

Proof. Let $E$ be $P$-extensive in $T, C$ compact in $T$ and $E \subset B C$. Let $t \in T$ such that $t C \subset P^{-1}$. Since we may assume that $e \in C$, we have $t \in P^{-1}$. Let $p \in P$, then $E \cap t^{-1} p P \neq \varnothing$; thus let $a \in E$ such that $a=t^{-1} p q$, where $q \in P$. We have also that $a=b c$, where $b \in B$ and $c \in C$. Thus $a t=b c t=b t c=b p_{0}^{-1}$, where $p_{0}^{-1}=t c \in t C \subset P^{-1}$. Therefore $b=a t p_{0}=p q t^{-1} t p_{0}=p q p_{0} \in p P$, whence $B \cap p P \neq \varnothing$. Thus $B$ is $P$-extensive. The conclusion follows from Lemma 2 , Theorem 2 and $[3$, 3.36]. This completes the proof.

\section{3. $P$-regional recurrence.}

Lemma 3. Let $x \in X$, then the following statements are pairwise equivalent:

(1) $x$ is $P$-regionally recurrent, i.e. if $U$ is a neighborhood of $x$, then there exists a $P$-extensive set $E$ in $T$ such that $r \in E$ implies $U \cap U r \neq \varnothing$.

(2) If $U$ is a neighborhood of $x$, then $U \cap U q P \neq \varnothing$ for each $q \in P$.

(3) If $U$ is a neighborhood of $x$, then $x \in \mathrm{Cl}(U q P)$ for each $q \in P$.

(4) If $U$ is a neighborhood of $x$ and if $t \in T$, then $U \cap U t q P \neq \varnothing$ for each $q \in P$. 
(5) If $U$ is a neighborhood of $x$ and if $t \in T$, then $x \in \mathrm{Cl}(U t q P)$ for each $q \in P$.

(6) If $U$ is a neighborhood of $x$, then $x T \subset \mathrm{Cl}(U q P)$ for each $q \in P$.

(7) If $U$ is a neighborhood of $x$, then $\mathrm{Cl}(x T) \subset \mathrm{Cl}(U q P)$ for each $g \in P$.

The proof is straightforward and is omitted.

Lemma 4. Let $x \in X$ and let $P$ be a replete semigroup in $T$. Let $x$ be $P$-regionally recurrent, and let $y \in \mathrm{Cl}(x T)$, then $y$ is P-regionally recurrent.

Proof. [3, 3.27].

TheOREM 4. Let $x \in X$, then each point of $P_{x}$ is P-regionally recurrent.

PRoof. Let $y \in P_{x}$, and let $U$ be a neighborhood of $y$, then $y \in \mathrm{Cl}(x p P) \subset \mathrm{Cl}(x P)$ for each $p \in P$. Let $p_{0} \in P$ such that $x p_{0} \in U$. Now $y \in \mathrm{Cl}\left(x p_{0} p P\right)$ for each $p \in P$; thus let $q_{p} \in p P$ such that $x p q_{p} \in U$. Let $E=\cup_{p \in P} p_{0}^{-1} p q_{p}$. We show that $E$ is $P$-extensive. Let $s \in P$, then there exists $q_{s} \in p_{0} P$ such that $p_{0}^{-1} s p_{s} \in E$. Thus $p_{0}^{-1} s q_{s} \in p_{0}^{-1} s\left(p_{0} P\right)$ $=s P$, and $E \cap s P \neq \varnothing$. We show that $y$ is $P$-regionally recurrent. Let $r \in E, r=p_{0}^{-1} p q_{p}$ for some $p \in P$; then since $x p_{0} \in U, x p_{0} r=x p_{0}\left(p_{0}^{-1} p q_{p}\right)$ $=x p q_{p} \in U r$. Since $x p q_{p} \in U$, we have $U \cap U r \neq \varnothing$. This completes the proof.

Although the following theorem is only a minor variation of $[3$, $7.15]$, it will be needed in the sequel, and had best be stated explicitly here.

Theorem 5. Let $T$ be generative, let $T$ be P-regionally recurrent and let $R$ be the set of all $P$-recurrent points of $X$. Then:

(1) If $X$ is metrizable, $R$ is an invariant residual $G_{\delta}$ set in $X$.

(2) If $X$ is complete, then $X=\mathrm{Cl}(R)$.

Proof. Use [3, 3.31] and [3, 6.09].

THEOREM 6. Let $A$ be the set of all $P$-regionally recurrent points of $X$, then

(1) $A$ is nonvacuous, closed, and invariant in $X$.

(2) If $T$ is generative and separable, then each point of $X-A$ is $P$-asymptotic to $A$.

Proof. (1) By $[3,6.34] P_{x} \neq \varnothing$ for each $x \in X$, and by Theorem 4 $A \supset P_{x}$, whence $A \neq \varnothing$. Let $y \in \mathrm{Cl}(A)$, and let $U$ be any open neighborhood of $y$, then $U \cap A \neq \varnothing$. Let $a \in U \cap A$, then since $U$ is open, 
$U$ is a neighborhood of $a$; and since $a$ is $P$-regionally recurrent, there exists a $P$-extensive set $E \subset T$ such that $r \in E$ implies $U r \cap U \neq \varnothing$. This, however, implies that $y$ is $P$-regionally recurrent, whence $y \in A$, and $A$ is closed.

Finally let $y \in A$, then, by Lemma $4, y t$ is $P$-regionally recurrent for each $t \in T$, whence $A$ is invariant.

(2) Let $x \in X-A$, then since $P_{x} \subset A$, the conclusion follows from $[1,2.12]$. This completes the proof.

Theorem 7. Let $X$ be a complete metric space, let $T$ be generative, then $T$ is $P$-regionally recurrent if and only if the set of all P-recurrent points of $X$ is dense in $X$.

Proof. We remark that if $x$ is $P$-recurrent then $x$ is $P$-regionally recurrent. The result then follows from Theorem 1, Theorem 6(1), and Theorem 5 .

TheOREM 8. Let $S$ be a closed syndetic subgroup of $T$ and let $T$ be locally compact. Then $S$ is $P \cap S$-regionally recurrent if and only if $T$ is $P$-regionally recurrent.

Proof. Let $S$ be $P \cap S$-regionally recurrent. Let $x \in X$, and let $U$ be a neighborhood of $x$; then there exists a $P \cap S$-extensive set $E \subset S$ such that $r \in E$ implies $U \cap U r \neq \varnothing$. However, by Theorem $2, E$ is $P$-extensive, whence $T$ is $P$-regionally recurrent.

Conversely, let $T$ be $P$-regionally recurrent, let $x \in X$, and let $U$ be a neighborhood of $x$. Then there exists an open neighborhood $U_{0}$ of $x$ and a compact symmetric neighborhood $V$ of $e$ in $T$ such that $U_{0} V \subset U$.

Now there exists $t \in T$ such that $t V \subset P$, whence $t \in P$. Let $p \in P \cap S$ $\subset P$, then $p t V P \subset P^{3} \subset P$ and $V(p t P) \cap S \subset p P \cap S$. We proceed inductively: there exists $p t p_{1} \in p t P$ such that $U_{0} \cap U_{0} p t p_{1} \neq \varnothing$. Thus there exists $x_{1} \in U_{0}$ such that $x_{1} p t p_{1} \in U_{0}$. There exists $U_{1}$, an open neighborhood of $x_{1}$, such that $U_{1} \subset U_{0}$ and $U_{1} p t p_{1} \subset U_{0}$.

At the $i$ th step: there exists $p t p_{i+1} \in p t P$ such that $U_{i} \cap U_{i} p t p_{i} \neq \varnothing$. Thus there exists $x_{i+1} \in U_{i}$ such that $x_{i+1} p t p_{i+1} \in U_{i}$. There exists $U_{i+1}$, an open neighborhood of $x_{i+1}$, such that $U_{i+1} \subset U_{i}$ and $U_{i+1} p p_{i+1}$ $\subset U_{i}$.

There exists $K \subset T$, compact, such that $T=S K$. For each $i=1$, $2, \cdots$, there exists $s_{i} \in S$ and $k_{i} \in K$ such that $p t p_{i}=s_{i} k_{i}$. By $[3$, $6.22]$ we can find $n$ positive integers, $i_{1}, i_{2}, \cdots, i_{n}(n \geqq 1)$ such that $i_{1}<i_{2}<\cdots<i_{n}$ and $k_{i_{1}} k_{i_{2}} \cdots k_{i_{n}} \in S V$. Since $U_{i_{j}} p t p_{i_{j}} \subset U_{i_{j-1}}$ for $j=2,3, \cdots, n$, and since $U_{i_{1} p t p_{i_{1}}} \subset U_{0}$, we have $U_{i_{2} p t p_{i_{2}} p t p_{i_{1}}}$ $\subset U_{i_{1}} p t p_{i_{1}} \subset U_{0}$, and in general $U_{i_{n}} p^{n} t^{n} p_{i_{n}} \cdots p_{i_{1}} \subset U_{0}$. Also $U_{i_{n}} \subset U_{0}$, 
whence $U_{i_{n}} p^{n} t^{n} p_{i_{n}} \cdots p_{i_{1}} \subset U_{0} p^{n} t^{n} p_{i_{n}} \cdots p_{i_{1}}$. Thus $U_{0} \cap U_{0} p^{n} t^{n} p_{i_{n}}$ $\cdots p_{i_{1}} \neq \varnothing$. Now $p^{n} t^{n} p_{i_{n}} \cdots p_{i_{1}}=s k_{i_{1}} k_{i_{2}} \cdots k_{i_{n}}$, where $s=s_{i_{1}} s_{i_{2}}$ $\cdots s_{i_{n}} \in S$. Select $s_{0} \in S$ and $v \in V$ such that $s_{0} v=k_{i_{1}} k_{i_{2}} \cdots k_{i_{n}}$; then $p^{n} t^{n} p_{i_{n}} \cdots p_{i_{1}}=s s_{0} v$.

Define $q=p^{n} t^{n} p_{i_{n}} \cdots p_{i_{1}} v^{-1}$. We observe that $q=s s_{0} \in S$ and that $q \in p t P V$, whence $q \in p t P V \cap S \subset p P \cap S$, and since $p \in P \cap S$, $q \in p(P \cap S)=p P \cap S$. Now $U_{0} v^{-1} \cap U_{0} p^{n} t^{n} p_{i_{n}} \cdots p_{i_{1}} v^{-1}=U_{0} v^{-1} \cap U_{0} q$ $\neq \varnothing$. But $U_{0} v^{-1} \subset U_{0} V \subset U$, and $U_{0} \subset U$, thus $U \cap U q \neq \varnothing$. Since $q \in p(P \cap S)$ and since $p \in P \cap S$ was arbitrary, we have that $U \cap U p(P \cap S) \neq \varnothing$ for each $p \in P \cap S$, whence, by Lemma $3, x$ is $P \cap S$-regionally recurrent. This completes the proof.

There is available another notion of recurrence parallel to compact asymptoticity $[1,2.19]$. We explore this notion briefly in the remainder of this section. Let $T$ be a noncompact group, then $E \subset T$ is said to be compactly extensive provided that for each compact set $K \subset T, E \cap(T-K) \neq \varnothing$. Let $x \in X$, then $x$ is said to be compactly recurrent provided that for each neighborhood $U$ of $x$ there exists a compactly extensive set $E \subset T$ such that $x E \subset U$.

Lemma 5. Let $T$ be a noncompact group and let $P$ be a replete semigroup in $T$. Let $K \subset T$ be compact, then there exists $q \in P$ such that $q P \cap K=\varnothing$.

Proof. We recall the basic assumption of $\$ 1$, that $P \neq T$. Assume the statement false, i.e. for each $q \in P, q P \cap K \neq \varnothing$. This implies that $P \subset P^{-1} K$, whence $T=P P^{-1} \subset K P^{-1} P^{-1} \subset K P^{-1}$, and $T=K P^{-1}$. Now since $K$ is compact, there exists $t \in T$ such that $t K^{-1} \subset P$, and then $T=t T=t T^{-1} \subset t K^{-1} P \subset P P \subset P$, and $P=T$. This contradiction completes the proof.

Theorem 9. Let $P$ be a replete semigroup in $T$, let $x \in X$ be P-recurrent, then $x$ is compactly recurrent.

Proof. Let $U$ be a neighborhood of $x$ and let $K \subset T$ be compact. Since $x$ is $P$-recurrent, there exists a $P$-extensive set $E \subset T$ such that $x E \subset U$. We show that $E$ is compactly extensive. By Lemma 5 there exists $q \in P$ such that $K \cap q P=\varnothing$, whence $(T-K) \supset q P$, and since $E \cap q P \neq \varnothing$, it follows that $E \cap(T-K) \neq \varnothing$. This completes the proof.

4. Applications. We define the $P$-center of the transformation group $(X, T, \pi)$ to be the greatest $T$-invariant subset $C_{P}$ of $X$ such that $T$ is $P$-regionally recurrent on $C_{P}$, i.e. such that $\left(C_{P}, T, \pi\right)$ is $P$-regionally recurrent. We remark that $C_{P}$ is the join of the class of all $T$ - 
invariant subsets of $X$ on which $T$ is $P$-regionally recurrent. The center of the transformation group $(X, T, \pi)$ is defined as in $[3,7.17]$.

Theorem 10. Let $C_{P}$ be the $P$-center of $(X, T, \pi)$, then the following are valid statements:

(1) $C_{P}$ is closed in $X$.

(2) $C_{P}$ is contained in the set of all $P$-regionally recurrent points of $X$.

(3) If $x$ is P-recurrent, $C_{P} \supset P_{x}$.

(4) $C_{P} \neq \varnothing$.

Proof. (1) and (2) are clear.

(3) Let $y \in P_{x}$. By Lemma $1, \mathrm{Cl}(x T)=P_{x}$. Let $U$ be a neighborhood of $y$ in $P_{x}$, then there exists $t \in T$ such that $x t \in U$. Let $V$ be a neighborhood of $x t$ in $P_{x}$ such that $V \subset U$ and let $V=V^{\prime} \cap P_{x}$, where $V^{\prime}$ is a neighborhood of $x t$ in $X$. By Theorem $1 x t$ is $P$-recurrent, thus there exists a $P$-extensive set $E \subset T$ such that $x t E \subset V^{\prime}$. Let $r \in E$, then $x \operatorname{tr} \in V^{\prime}$, and since $x t \in V^{\prime}, x t r \in V^{\prime} r$. Also since $x t, x t r \in P_{x}=\mathrm{Cl}(x T)$, we have $x t \in V^{\prime} \cap P_{x}=V$, and $x t r \in V^{\prime} r \cap P_{x}=V r$, whence $\varnothing \neq V \cap V r$ CUnUr. Thus $P_{x}$ is a $T$-invariant subset of $X$ on which $T$ is $P$ regionally recurrent, and $C_{P} \supset P_{x}$.

(4) We remark that $[3,2.22]$ guarantees the existence of a recurrent point $x \in X$. It is clear that $x$ is recurrent if and only if $x$ is $P$-recurrent for each replete semigroup $P$ in $T$. The result then follows from (3) above and $[3,6.34]$. This completes the proof.

Another approach to the $P$-center is the following: if $Y$ is an invariant subset of $X$, define $Y^{*}$ to be the set of all points of $Y$ at which the transformation group $(Y, T, \pi)$ is $P$-regionally recurrent. For each ordinal $\alpha$ define $P^{R} \alpha$ as follows:

(1) $P^{R_{0}}=X$.

(2) If $\alpha$ is a succession ordinal, then $P^{R} \alpha=\left(P^{R} \alpha-1\right)^{*}$.

(3) If $\alpha$ is a limiting ordinal, then $P^{R} \alpha=\bigcap_{\beta<\alpha} P^{R} \beta$.

Then:

(1) There exists a least ordinal $\gamma$ such that $P^{R} \gamma=P^{R} \alpha$ for all $\alpha>\gamma$.

(2) If $X$ is second countable, then $\gamma$ is countable.

(3) $P^{R} \gamma=C_{P}$.

We also remark that if $C$ is the center of $(X, T, \pi)$ and if $\rho$ is the class of all replete semigroups in $T$, then $C \subset \cap_{P \in \mathcal{P}} C_{P}$.

TheOREM 11. Let $X$ be a complete metric space and let $T$ be generative, then the $P$-center of $(X, T, \pi)$ coincides with the closure of the set of all $P$-recurrent points of $X$.

Proof. Let $R_{P}$ be the collection of all $P$-recurrent points of $X$ and let $R_{C P}$ be the collection of all $P$-recurrent points of $C_{P}$. By Theorem 
6, $C_{P}=\mathrm{Cl}\left(R_{C P}\right)$. We show $R_{P}=R_{C P}$. Let $x \in R_{C P}$ and let $V$ be a neighborhood of $x$ in $C_{P}$, then $V=V^{\prime} \cap C_{P}$, where $V^{\prime}$ is a neighborhood of $x$ in $X$. Since $x \in R_{C P}$, there exists a $P$-extensive set $E$ in $T$ such that $x E \subset V \subset V^{\prime}$, whence $x \in R_{P}$. Conversely, let $x \in R_{P}$, then since $x$ is $P$-recurrent, $T$ is pointwise $P$-regionally recurrent on $x T$, i.e. $(x T, T, \pi)$ is $P$-regionally recurrent. Hence $x T \subset C_{P}$, and $x \in R_{C P}$. Thus $R_{P}=R_{C P}$, and this completes the proof.

Let $S$ be a subset of $T$ and let $M \subset X$. The set $M$ is called $S$-incompressible provided $M S \subset M$ implies $M S=M$. We now explore the relation between $P$-recurrence and incompressibility.

ThEOREM 12. $T$ is pointwise P-recurrent if and only if each closed set in $X$ is $p P$-incompressible for each $p \in P$.

Proof. Let $T$ be pointwise $P$-recurrent, let $M \subset X$ be closed and let $p \in P$ such that $M p P \subset M$. Let $x \in M$, then $x p P \subset M p P \subset M$. We remark that by Lemma $1, x \in P_{x}=\mathrm{Cl}(x P)$ and that by $[3,6.34]$ $P_{x}$ is invariant, consequently $x \in P_{x}=P_{x} p^{2} P=([\mathrm{Cl}(x P)] p) p P$ $=[\mathrm{Cl}(x p P)] p P \subset[\mathrm{Cl}(M)] p P=M p P$, whence $x \in M p P$ and $M \subset M p P$. Thus $M=M p P$.

Conversely, let each closed set of $X$ be $p P$-incompressible for each $p \in P$. Let $x \in X$, let $p \in P$ and let $M=\mathrm{Cl}(x \cup x p P)$. Then $M p P$ $\subset \mathrm{Cl}(x p P) \subset M$ and by hypothesis $M p P=\mathrm{Cl}(x p P)=M$. It follows that $x \in \mathrm{Cl}(x p P)$ for each $p \in P$ and by Lemma $1, x$ is $P$-recurrent. This completes the proof.

Theorem 13. $T$ is pointwise $P$-recurrent if and only if each open set in $X$ is $p^{-1} P^{-1}$-incompressible for each $p \in P$.

Proof. Let $T$ be pointwise $P$-recurrent. We remark that by Lemma $1, x T \subset U P^{-1}$ for each neighborhood $U$ of $x$, and thus $x T=x T p^{-1}$ $\subset U p^{-1} P^{-1}$ for each $p \in P$.

Let $M$ be an open set in $X$ and let $p \in P$ such that $M p^{-1} P^{-1} \subset M$. Let $x \in M$, then there exists a neighborhood $U$ of $x$ such that $U \subset M$. Thus $x \in x T \subset U p^{-1} P^{-1} \subset M p^{-1} P^{-1}$, and $M \subset M p^{-1} P^{-1}$, whence $M=M P^{-1} P^{-1}$.

Conversely, let each open set of $X$ be $p^{-1} P^{-1}$-incompressible. Let $t \in T$, then since $T=P^{-1} P, t=r^{-1} q$, where $r, q \in P$; also let $p \in P$ and define $M=U t \cup U P^{-1}$. Then $M q^{-1} p^{-1} P^{-1}=U t q^{-1} p^{-1} P^{-1}$ $\cup U q^{-1} p^{-1} P^{-1} P^{-1} \subset U r^{-1} p^{-1} P^{-1} \cup U q^{-1} p^{-1} P^{-1} \subset U P^{-1} \subset M$, and since by hypothesis $M q^{-1} p^{-1} P^{-1}=M$ it follows that $M q^{-1} p^{-1} P^{-1}=U P^{-1}$ $=M$, whence $U t \subset U P^{-1}$. Thus $x t \in U t \subset U P^{-1}$ for each $t \in T$ and $x T \subset U P^{-1}$. By Lemma 1, $x$ is $P$-recurrent. This completes the proof. 
Corollary. Let $P$ be a replete semigroup in $T$ and let each $\{$ closed \{open $\}$ set in $X$ be $\{p P$-incompressible $\}\left\{p^{-1} P^{-1}\right.$-incompressible $\}$ for each $p \in P$, then $C_{P}=X$.

ThEOREM 14. Let $X$ be a compact metric space, let $T$ be generative and let $P$ be a replete semigroup in $T$. Let $C$ be the center of $X$ and $C_{P}$ be the $P$-center. For $B \subset X$ and $x \in X$ define $P(x, B)$ as in [2, Definition $2.1]$, and let $N(B)$ be defined to be any open set containing $B$. Then if $x \in X, P\left(x, N\left(C_{P}\right)\right)=P(x, N(C))=1$.

Proof. Let $R$ be the set of all recurrent points of $X$, let $R_{P}$ be the set of all $P$-recurrent points of $X$, and let $A$ be the minimal center of attraction of $X[2$, Definition 3.4]. If $x$ is recurrent, then clearly $x$ is $P$-recurrent, hence $R_{P} \supset R$ and by [2, Theorem 4.2], $R \supset A$. Furthermore by Theorem $11, C_{P}=\mathrm{Cl}\left(R_{P}\right)$ and $C=\mathrm{Cl}(R)$, whence $C_{P} \supset C \supset A$ and it follows by $\left[2\right.$, Theorem 3.6] that $P\left(x, N\left(C_{P}\right)\right)$ $=P(x, N(C))=1$. This completes the proof.

\section{BIBLIOGRAPHY}

1. John D. Baum, Asymptoticity in topological dynamics, Trans. Amer. Math. Soc. vol. 77 (1954) pp. 506-519.

2. R. R. Bernard, Probability in dynamical transformation groups, Duke Math. J. vol. 18 (1951) pp. 307-319.

3. W. H. Gottschalk and G. A. Hedlund, Topological dynamics, Providence, 1955.

Oberlin College 